\title{
Adaptive Cluster Head Selection Scheme for High Mobility Based IEEE 802.15.6 Wireless Body Area Networks
}

\author{
Anthony Mile, George Okeyo, Ann Kibe \\ School of Computing and Information Technology, Jomo Kenyatta University of Agriculture and Technology, Nairobi, Kenya \\ Email: Mileanthony@yahoo.com, gokeyo@jkuat.ac.ke, annkibe@icsit.jkuat.ac.ke
}

How to cite this paper: Mile, A., Okeyo, G. and Kibe, A. (2018) Adaptive Cluster Head Selection Scheme for High Mobility Based IEEE 802.15.6 Wireless Body Area Networks. Journal of Sensor Technology, 8 , 35-47.

https://doi.org/10.4236/jst.2018.82003

Received: May 4, 2018

Accepted: June 11, 2018

Published: June 14, 2018

Copyright ( 92018 by authors and Scientific Research Publishing Inc. This work is licensed under the Creative Commons Attribution International License (CC BY 4.0).

http://creativecommons.org/licenses/by/4.0/

(c) (i) Open Access

\begin{abstract}
Due to the development in the field of Wireless Sensor Networks (WSNs), its major application, Wireless Body Area Network (WBAN) has presently become a major area of interest for the developers and researchers. Efficient sensor nodes data collection is the key feature of any effective wireless body area network. Prioritizing nodes and cluster head selection schemes plays an important role in WBAN. Human body exhibits postural mobility which affects distances and connections between different sensor nodes. In this context, we propose maximum consensus based cluster head selection scheme, which allows cluster head selection by using Link State. Nodal priority through transmission power is also introduced to make WBAN more effective. This scheme results in reduced mean power consumption and also reduces network delay. A comparison with IEEE 802.15.6 based CSMA/CA protocol with different locations of cluster head is presented in this paper. These results show that our proposed scheme outperforms Random Cluster head selection, Fixed Cluster head at head, Foot and Belly positions in terms of mean power consumption, network delay, network throughput and bandwidth efficiency.
\end{abstract}

\section{Keywords}

Adaptive Cluster Head, CSMA/CA, LST, Nodal Priority, WBAN

\section{Introduction}

A wireless sensor network (WSN) consists of sensor nodes to examine and monitor environmental factors such as temperature, sound etc. and send their data to the base station as discussed in [1] [2] [3]. Wireless Body Area Network (WBAN) is a special application of WSN to monitor variables remotely. A 
WBAN is a special purpose sensor network which is developed to manage and communicate between various medical sensors, which are positioned inside and outside the human body [4]. A special example of WBAN is presented in Figure 1. In tier 1, these sensors, sense body temperature, heart beat rate, pulse rate and other required data and send it to personal coordinator through ZigBee or Bluetooth. The personal server or personal coordinator in tier 2 sends these values to medical server through internet. The health care provider (doctor) sitting in tier 3 examines the results and gives precautions and medical treatments to the patient [5].

Human beings generally change their postures as shown in Figure 2. As the human body moves, the wireless connectivity amongst the nodes also varies. In this situation, the data cluster head has to be changed and adjusted as distance between sensor nodes varies. Another thing, which is very important for a cluster head, is its accessibility by all neighboring nodes. This would ensure that each node sends its data to the cluster head, which will ultimately increase the reliability of WBAN.

Our proposed scheme is called Adaptive Cluster Head Selection Scheme with Nodal Priority for wireless body area networks, in which we are selecting a cluster head on the basis of link state. We have used Omnet++ with Mixim framework, which makes simulation, more realistic and reliable [6]. MoBAN mobility model, which is discussed in [7] and [15], is also used to establish postures for human body.

The main objective of proposed scheme is to make WBAN more reliable and energy efficient by introducing idea of acknowledgement and adaptive cluster head selection in CSMA/CA protocol. By introducing acknowledgements, we can ensure that the two nodes are connected and data transmission is taking place successfully. There is no such mechanism in IEEE 802.15.6 based CSMA/CA protocol. Another approach that we have introduced is the idea of adaptive cluster head selection with nodal priority in CSMA/CA, protocol which

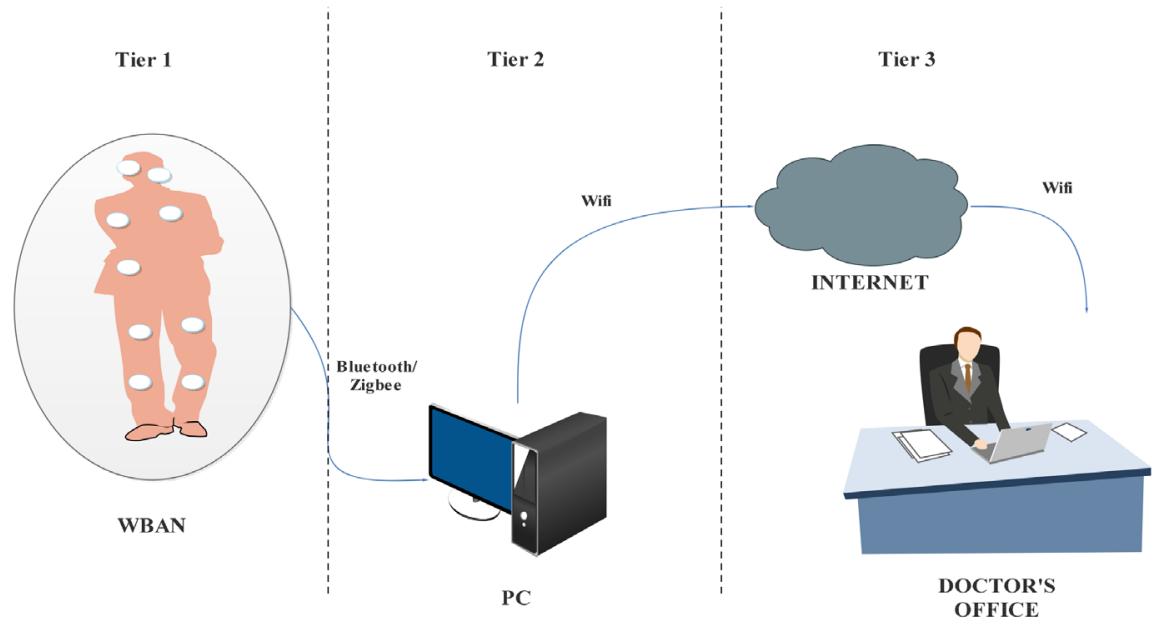

Figure 1. Wireless body area network. 


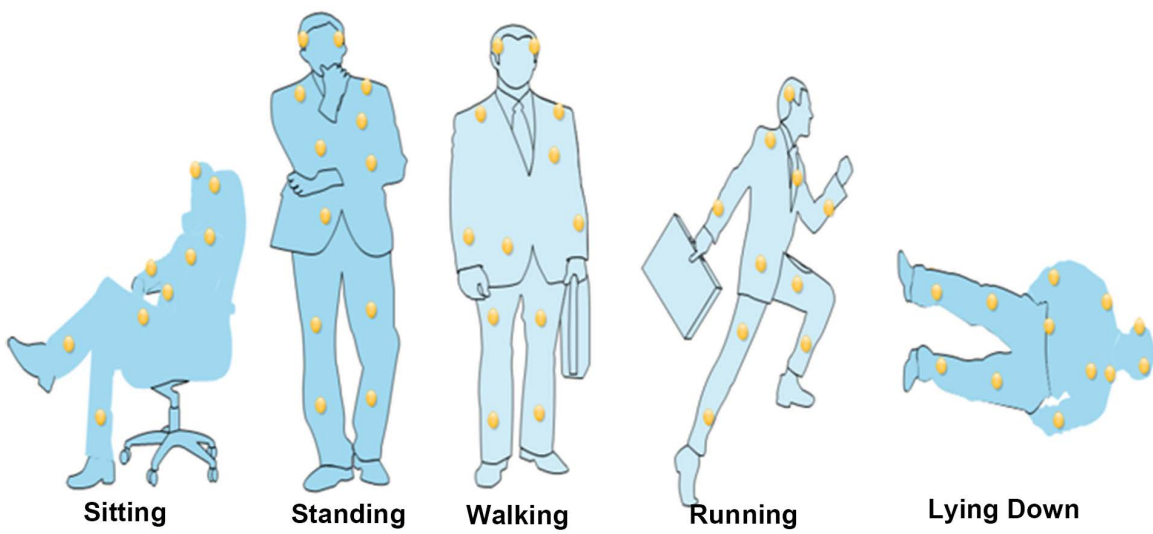

Figure 2. Human body posture.

reduces data loss and also increases network throughput, thus ultimately making CSMA/CA more efficient and effective.

The rest of the paper is organized as follows. In Section 2, related work is discussed. In Section 3, we discuss the proposed scheme while in Section 4, we discuss the performance parameters. Section 5 contains results and analysis and conclusion.

\section{Related Work}

Nowadays, smart healthcare services are one of the most preferable demands of our society. Our work comprises of using the proper MAC scheme for assigning nodal priority. The commonly used MAC layer protocols include S-MAC, B-MAC, LMAC, Wise MAC and IEEE 802.15.6 based CSMA/CA Protocol.

Sensor MAC protocol is one of the energy efficient MAC protocols. According to [8] SMAC reduces energy consumption by avoiding collisions, idle listening, overhearing, and minimizing control packet overheads. Periodic sleeping is an integral working component of SMAC, and according to conditions a node will go to sleep state if there is no data transmission or reception. During the sleep state, the node turns its radio off, and sets a timer for switching to "awake" status later.

B-MAC, commonly called Berkeley MAC protocol is an LPL (Low power listening) based protocol discussed in [9]. Its objective is to assign greater sleep intervals to nodes for optimum network lifetime and waking up after regular intervals to check for ongoing data communication.

Lightweight Medium Access protocol also known as LMAC is a TDMA based protocol. According to [10] and [11], LMAC network is self-organizing in terms of slot assignment and synchronization.

Wise MAC is also an energy efficient MAC protocol, which is defined in [12] and [13]. Wise MAC is based upon non persistent CSMA and reduces power consumption by using preamble sampling and by reducing idle listening.

The MAC layer protocol, which we chose to enhance, is the hybrid IEEE 802.15.6 based CSMA/CA interference Mitigation model [14]. This CSMA/CA based protocol proposed a hybrid WBAN interference mitigation model based 
on CSMA/CA Contention Window (CW) approach and User Priority (UP) queues. The reason for enhancing this current scheme is because the techniques proposed in the paper enables allocation of high priority to emergency critical sensor data and the rest of data is given lower priorities accordingly [14]. In the IEEE 802.15.6 based CSMA/CA Protocol, in which the node sets a back off counter between 1 and contention window size. If the channel is idle, the node will decrement the back off counter by one for each idle CSMA slot. When the back off counter becomes zero, the node will transmit the data or frame. If the channel is found busy, the node will lock its back off counter until the channel becomes idle. In the existing study [14], a counter which counts the number of failures is presented. Two cases are presented, the first one in which the number of failures is odd, then Contention Window (CW) size will remain unchanged and if the number of failures is even, then CW size will be doubled. After successful data transmission, $\mathrm{CW}$ is set to initial CW. The enhanced hybrid model also takes into account high mobility IEEE 802.15.6 making it a perfect baseline for this study.

\section{Proposed Scheme}

Easily accessible cluster head for data packets is very important for wireless body area network. As we know that in WBAN, distances and connectivity between different nodes vary according to the posture, as shown in Figure 3 and Figure 4. Using fixed cluster head has no significance because other nodes may or may not access that particular node. So, it is required to use cluster head which keeps changing throughout network lifetime. Routing table is commonly used to select a random cluster head for data packets, as done in Omnet++/Mixim, but depending on the routing table only is not a very effective technique. The reason is that a random cluster head may or may not be accessible by other nodes, which will ultimately create problems in WBAN, because information from every node will help doctors to treat patient in an effective way. So, information gathering from every node is a key feature of an effective WBAN.

In such situation, adaptive cluster head for data packets, which keeps changing depending upon the number of connections that each node holds thus contributing to enhance performance of the overall network. Introducing priority for nodes will further make this scheme more efficient. So, we propose adaptive cluster head selection with nodal priority. Explanation of our proposed scheme is done in this section.

Link state table is a very important feature of our scheme. This table actually contains information about number of connections that each node holds. This table is maintained at each node. The complete procedure for updating this table and selecting adaptive cluster head on the basis of LST is illustrated through Flow Chart 1. According to Flow Chart 1, after initializing important parameters like CWmin count, backoff_count, channel idleness will be checked, if idle, then backoff_count will be decremented until it becomes zero. Consequently, each node will broadcast control packet, upon the reception of control packets 

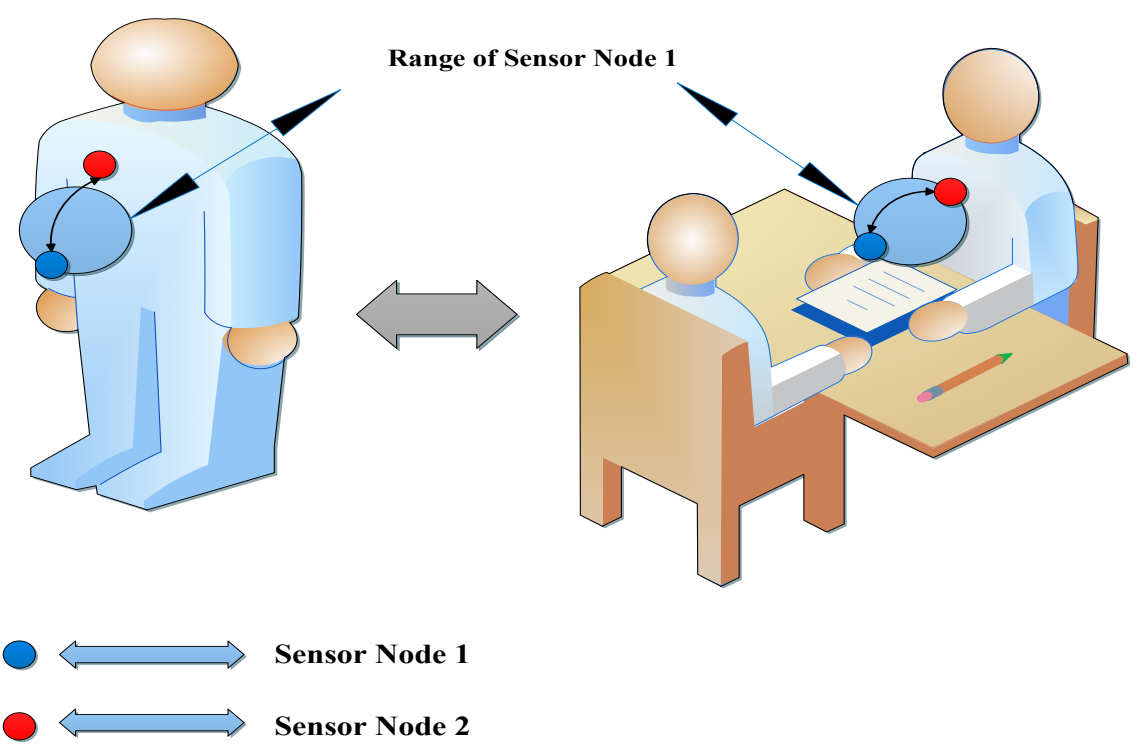

Figure 3. Illustration of range changing sensor nodes with postural changes.

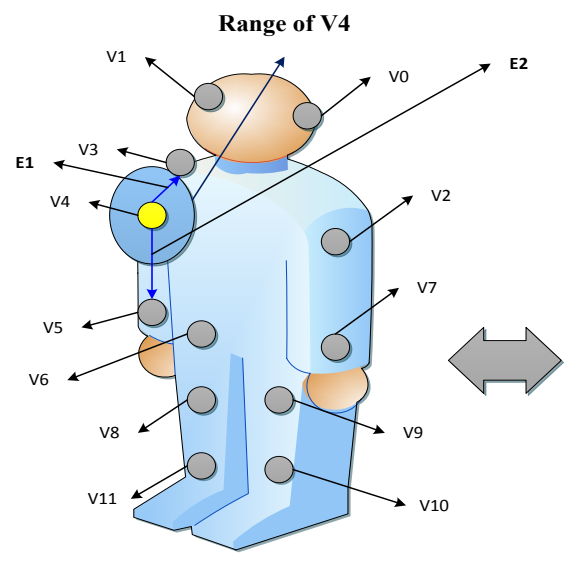

$E(t)=\{(V 4, V 3)\}$

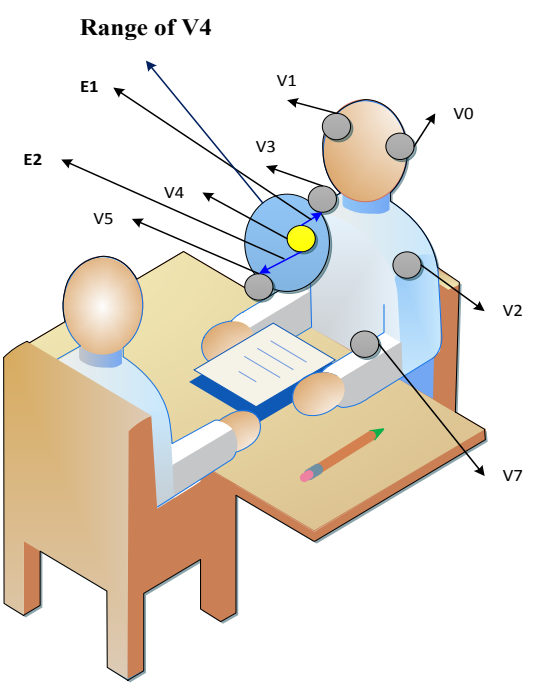

$E(t)=\{(V 4, V 3),(V 4, V 5)\}$

Figure 4. Illustration of variation in No. of connections with postural changes.

from other nodes, each node will acknowledge the sender that the control packet has been received. Link State Table (LST) will be updated (Incrementing No. of Links) after reception of acknowledgement otherwise it will remain unchanged. The node having maximum value in LST shows that it has maximum number of connections as compared to other nodes for current posture. So, there will be a competition between all sensors nodes to become "Cluster Head" on the basis of value stored in their LST. Node with maximum value in LST will become cluster head for other nodes. Power of those nodes which are not directly connected to the cluster head, will be increased, so that, each node can send its data to the cluster head. After that, data will be transmitted, if transmission is not successful, then number of failures will be counted, if odd, then contention Window 


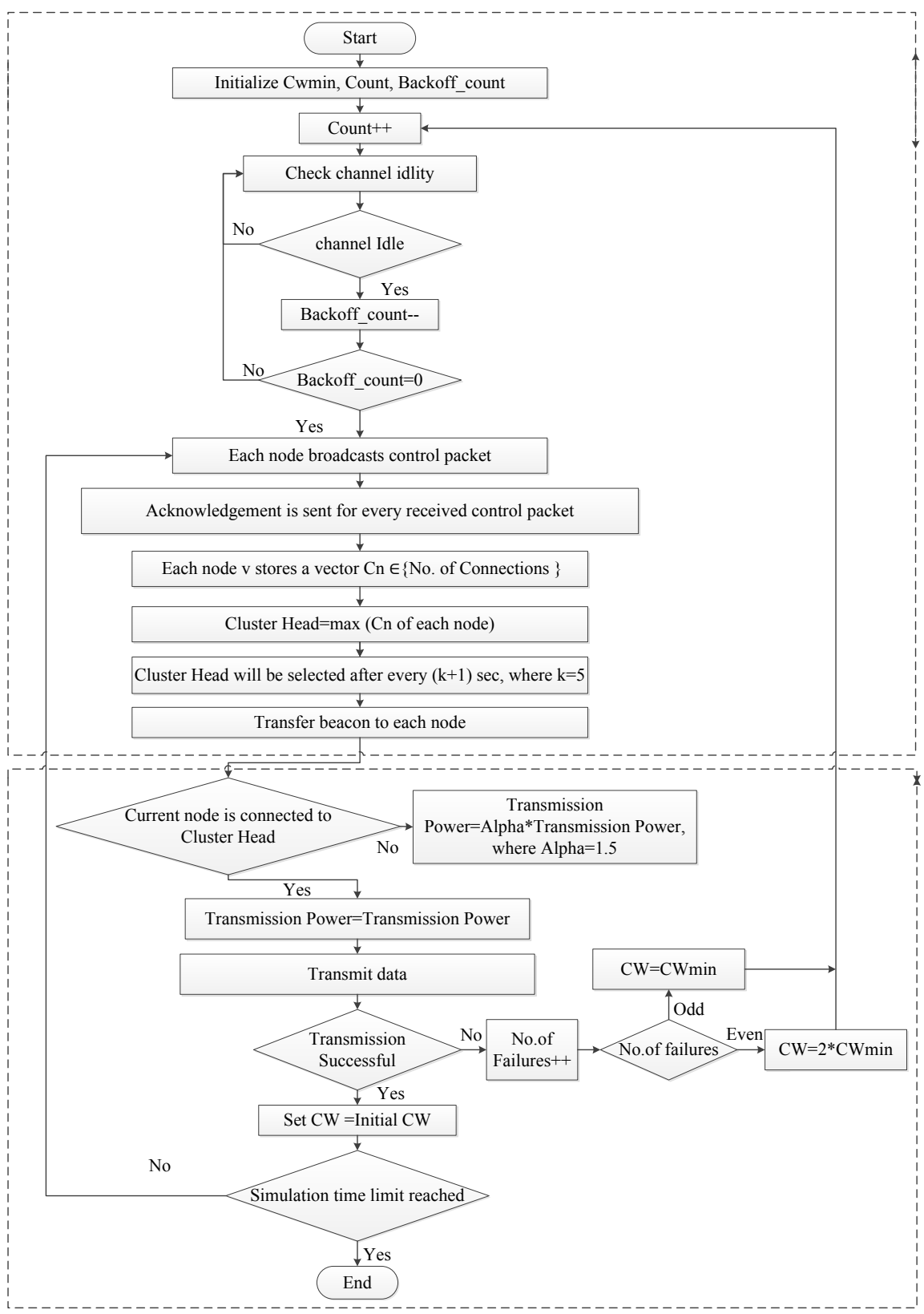

Flow Chart 1. Proposed scheme.

size will remain same otherwise it will be doubled. The reason is that even number of failures would confirm that there is a problem in contention window size, which stops transmission.

A simple example for cluster head selection is given in Figure 5. According to Figure 5, at time $t_{k+1}$, node 5 will become cluster head node because of holding maximum no. of connections. At $t_{k+21}$, node 3 , will become cluster head.

\section{Performance Parameters}

User priority list is presented in Table 1. Important notations and parameters list is given in Table 2 and Table 3 respectively. 


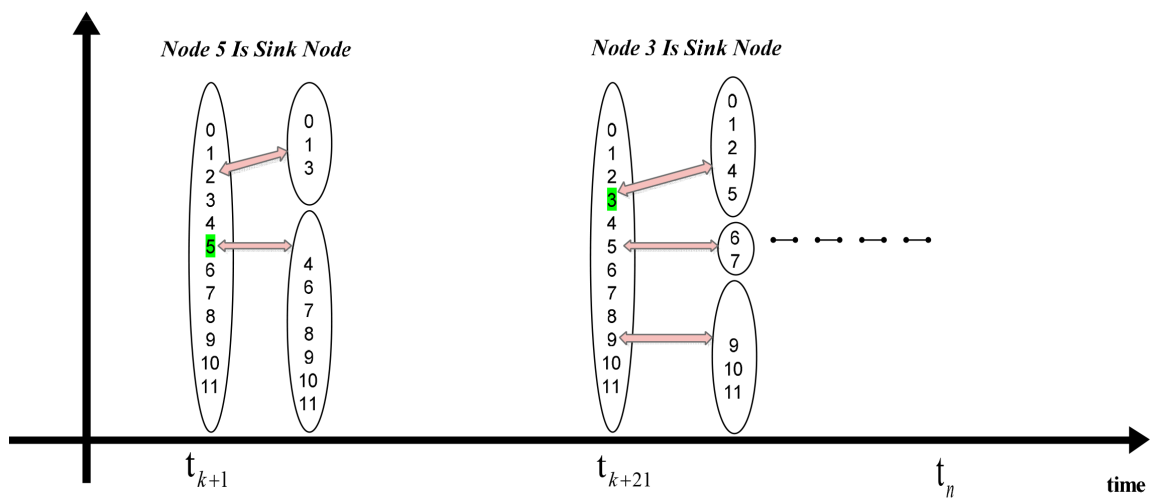

Figure 5. Example related to proposed scheme.

Table 1. Priority list.

\begin{tabular}{ccc}
\hline User & Transmission Power $(\mathrm{mW})$ & Priority \\
\hline 0 & 1 & 1 st \\
1 & 0.96 & 2 nd \\
2 & 0.92 & 3 rd \\
3 & 0.88 & 4 th \\
4 & 0.84 & 5 th \\
5 & 0.80 & 6 th \\
6 & 0.76 & 7 th \\
7 & 0.72 & 8 th \\
8 & 0.68 & 9 th \\
9 & 0.64 & 10 th \\
10 & 0.60 & 11 th \\
11 & 0.56 & 12 th
\end{tabular}

Table 2. Notations used in Equations.

\begin{tabular}{cc}
\hline$T_{p}$ & Preamble transmission time \\
$R_{s}$ & Preamble transmission symbol rate \\
$T_{P H Y}$ & PHY layer header transmission time \\
$R_{h d r}$ & Header rate of PHY layer \\
$R_{D A T A}$ & Transmission rate of data \\
$T_{s}$ & Slot length \\
$T_{p S I F S}$ & Short inter-frame spacing time \\
$M H R$ & Mac header \\
$F T R$ & Mac footer \\
$\tau$ & Propagation time \\
$x$ & Payload Size \\
$C W_{\text {min }}$ & Contention Window \\
\hline
\end{tabular}


Table 3. Simulation parameters.

\begin{tabular}{|c|c|}
\hline Parameter Name & Value \\
\hline Saturation & $-90 d B_{m}$ \\
\hline Alpha & 3 \\
\hline Carrier Frequency & $2.412 \mathrm{GHz}$ \\
\hline Time $R_{x}$ to $T_{x}$ & $0.00021 \mathrm{~s}$ \\
\hline Time $R_{x}$ to Sleep & $0.000031 \mathrm{~s}$ \\
\hline Time $T_{x}$ to $R_{x}$ & $0.00012 \mathrm{~s}$ \\
\hline Time $T_{x}$ to Sleep & $0.000032 \mathrm{~s}$ \\
\hline Time Sleep to $R_{x}$ & $0.000102 \mathrm{~s}$ \\
\hline Time Sleep to $T_{x}$ & $0.000203 \mathrm{~s}$ \\
\hline Battery Voltage & $3.3 \mathrm{~V}$ \\
\hline$T_{p M I F S}$ & $20 \mu s$ \\
\hline$T_{p S I F S}$ & $50 \mu s$ \\
\hline$T_{p}$ & $88 \mathrm{bit} / R_{s}$ \\
\hline$\tau$ & $1 \mu s$ \\
\hline$T_{P H Y}$ & 31 bits/ $R_{h d r}$ \\
\hline Mac Queue Length & 5 \\
\hline Slot Duration & $0.00035 \mathrm{~s}$ \\
\hline $\operatorname{Max} T_{x}$ Attempts & 14 \\
\hline$R_{D A T A}$ & $187,500 \mathrm{bps}$ \\
\hline$C W_{\min }$ & 16 \\
\hline$T_{x}$ Power & $1 \mathrm{~mW}$ \\
\hline$M H R$ & 56 \\
\hline$F T R$ & 16 \\
\hline$R_{s}$ & 187,500 \\
\hline$P_{\text {delay }}$ & 0.000001 \\
\hline$x$ & 2000 \\
\hline
\end{tabular}

Formula used to calculate delay normally, is given in Equation (1) is calculated in [15] as follows;

$$
\operatorname{Delay}(x)=T_{\text {avg_backoff }}+T_{\text {DATA }}+T_{\text {iack }}+2 T_{\text {pSIFS }}+2 \tau
$$

The average back off time can be found as shown in Equation (2);

$$
T_{\text {avg_backoff }}=\frac{C W_{\min } \times T_{s}}{2}
$$

The transmission time of data is $T_{D A T A}$ and can be obtained as in Equation (3);

$$
T_{\text {DATA }}=T_{p}+T_{P H Y}+\frac{(M H R+x+F T R)}{R_{D A T A}}
$$

The transmission time of immediate acknowledgement can be obtained as in Equation (4); 


$$
T_{\text {iack }}=T_{P}+T_{P H Y}+\frac{((M H R+F T R) * n)}{R_{D A T A}}
$$

Maximum Throughput (MT) of network is directly related to overhead.

The MT is defined as the ratio of payload size $(x)$ to the total transmission delay per payload size Delay(x), as given below in Equation (5) [15];

$$
M T=\frac{x}{\operatorname{Delay}(x)}
$$

Maximum Throughput $(M T)$ in case of immediate acknowledgement can be found by using Equation (6) [3];

$$
M T_{\text {iack }}=\frac{x}{\left(T_{\text {avg_backoff }}+T_{D A T A}+T_{\text {iack }}+2 T_{p S I F S}+2 \tau\right)}
$$

The bandwidth efficiency is inversely proportional to the basic data rate as in Equation (7);

$$
\rho=\frac{M T}{R_{D A T A}}
$$

\section{Performance Analysis}

Figure 6 shows delay versus time comparison between fixed cluster head, random cluster head selection scheme, Adaptive cluster head selection scheme and adaptive cluster head selection scheme with priority for mobile network. In these results, lower delay is achieved in adaptive cluster head selection scheme with priority.

Figure 7 shows throughput versus time comparison between fixed cluster head, random cluster head selection scheme, adaptive cluster head selection scheme and adaptive cluster head selection scheme with priority for mobile network. As depicted from results, higher throughput is achieved in adaptive cluster head selection scheme with priority.

Figure 8 shows bandwidth efficiency versus time comparison between fixed cluster head, random cluster head selection scheme, adaptive cluster head selection scheme and adaptive cluster head selection scheme with priority for mobile network. As seen from results, higher bandwidth efficiency is achieved in adaptive cluster head selection scheme with priority.

Figure 9 shows power consumption comparison between fixed cluster head, random cluster head selection scheme, adaptive cluster head selection scheme and adaptive cluster head selection scheme with priority for mobile network. As shown from results, low power consumption is achieved in adaptive cluster head selection scheme with priority.

As the cluster head node has maximum number of connections available in Adaptive cluster head selection scheme, that's why it is easier for other nodes to access cluster head, which results in better performance. Adding priority further enhances its performance. While in random cluster head selection scheme, 


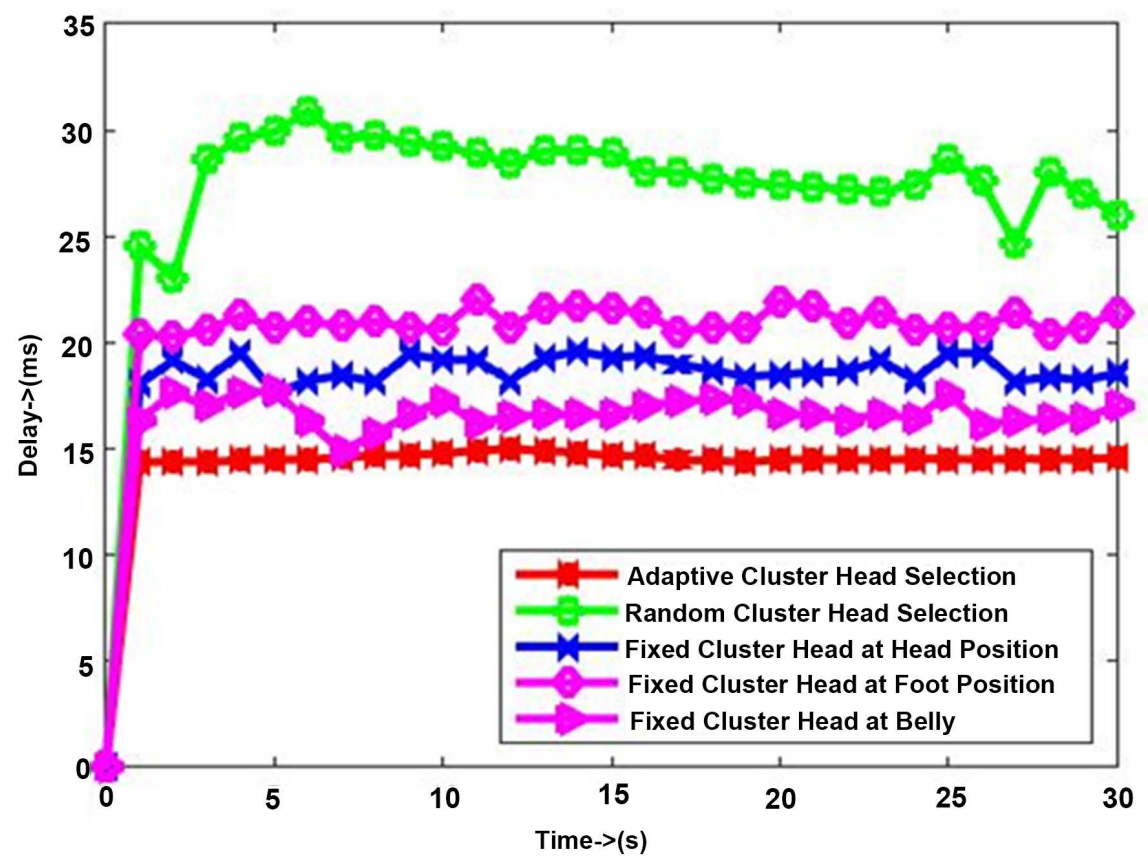

Figure 6. Mobile network delay on different cluster head.

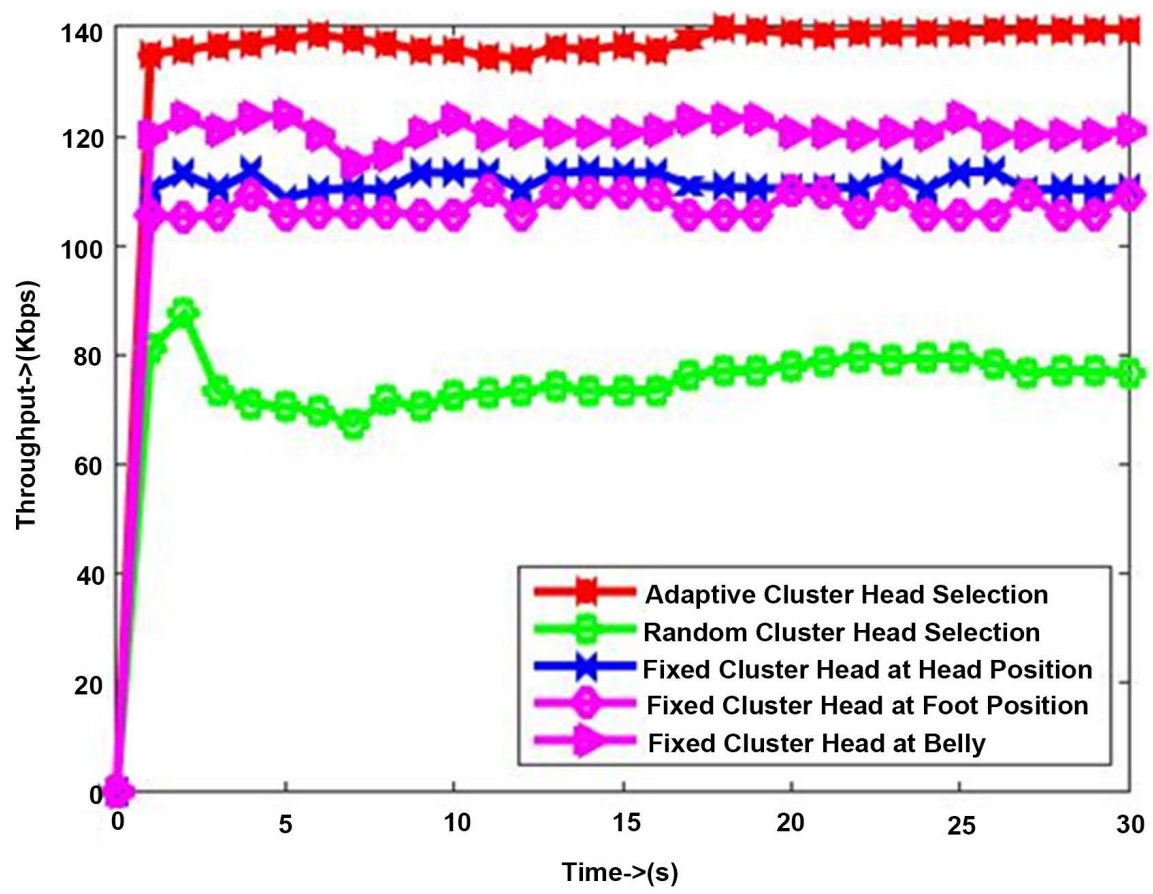

Figure 7. Mobile network throughput on different cluster head.

destination node may or may not have enough number of connections to accommodate nodes, which results in more delay, more power consumption and lower throughput.

Fixed cluster head selection schemes show better results as compared to random cluster head selection schemes, because in this case, randomly selected cluster head is not easily accessible as compared to fixed cluster heads for 


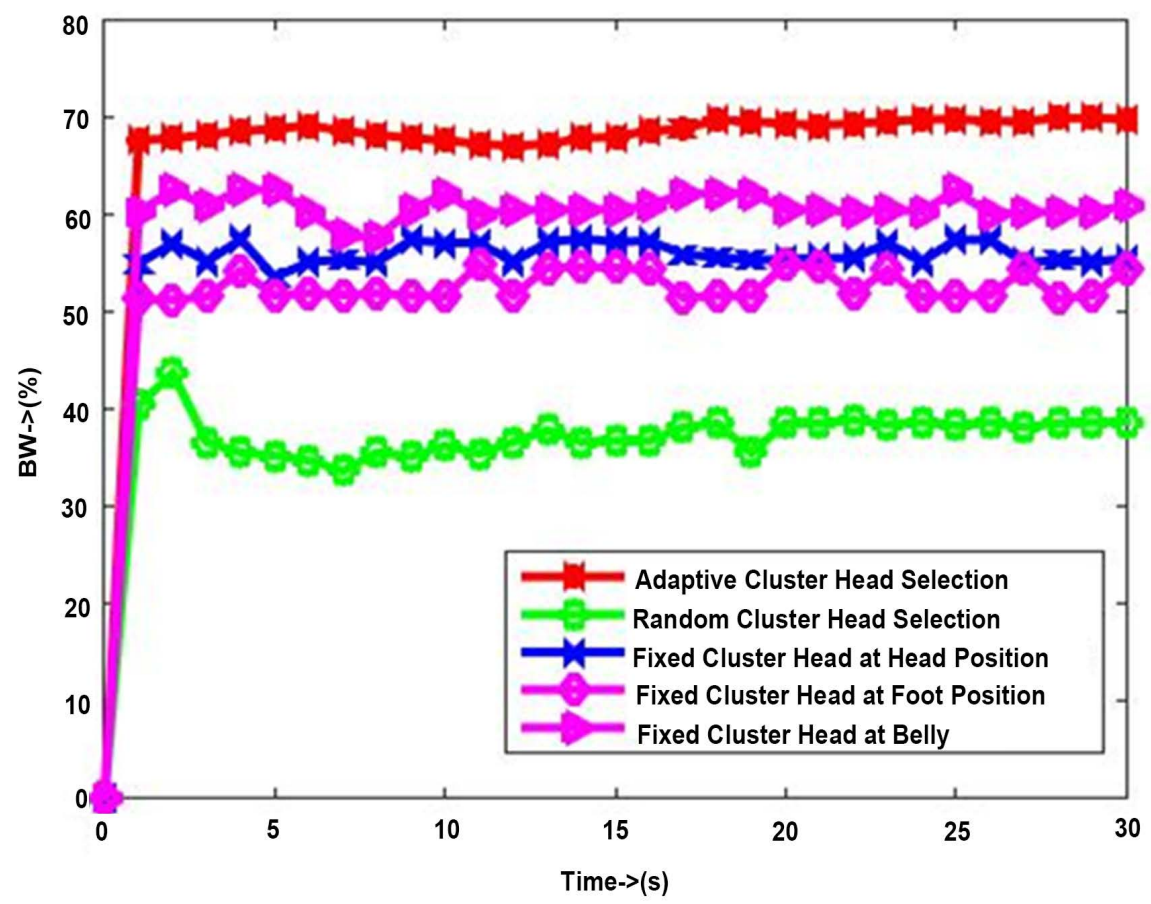

Figure 8. Mobile network bandwidth on different cluster head.
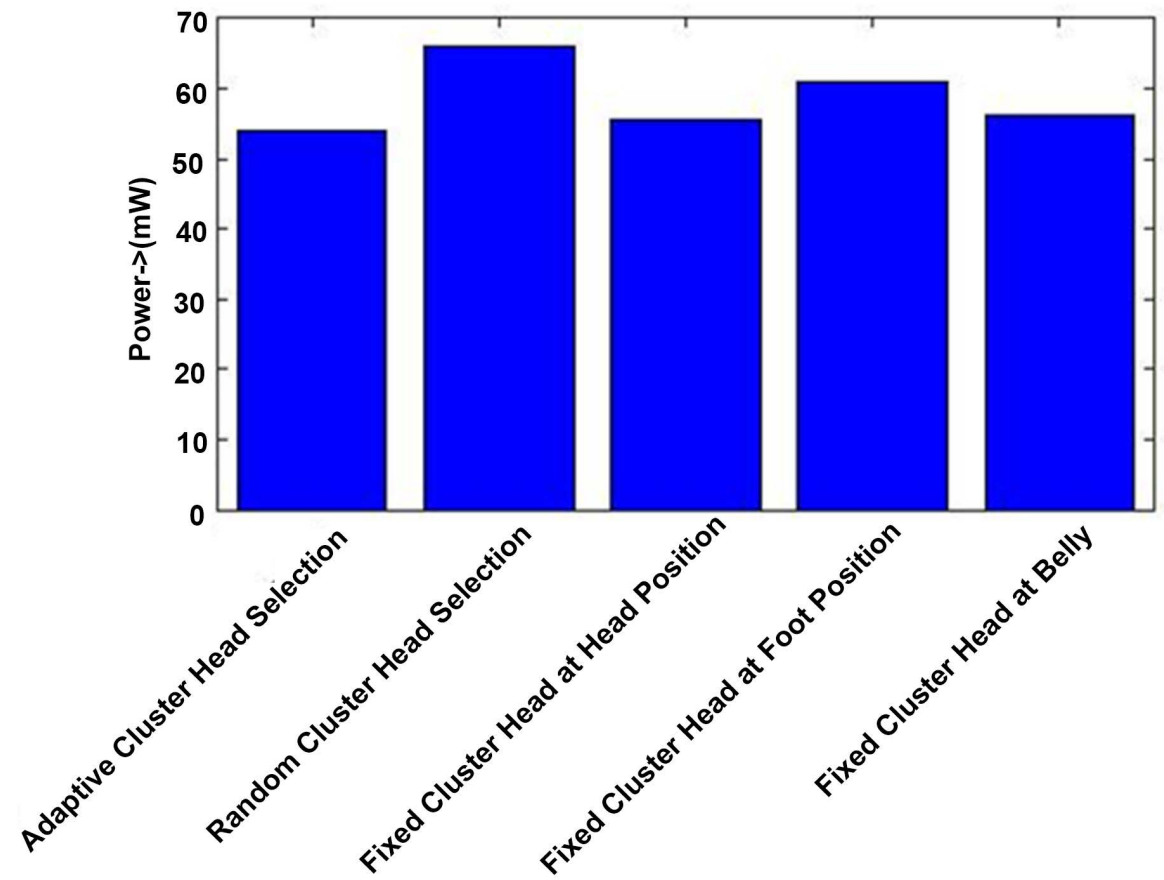

Figure 9. Mobile network power consumption on different cluster head.

current posture. Another important thing which is depicted from these results is that adaptive cluster head selection scheme and adaptive cluster head selection scheme with priority show steady behavior after 20 seconds, because at that time, network has adopted a cluster head, which gives ultimate performance. This behavior is lacking in random cluster head selection scheme. 


\section{Conclusions}

Inappropriate cluster head selection scheme is one of the major factors of data loss in WBAN. Adaptive cluster head selection scheme with nodal priority is proposed in this paper. This scheme is based upon adaptive cluster head selection using link state table which helps in reducing network delay, mean power consumption and increasing network throughput. In the proposed scheme, cluster head for data packet will be adaptive to the variation in number of connections that each node holds. The number of connections for every node is present in link state table.

The node having maximum number of connections will be selected as the cluster head and other nodes will consequently communicate with this node. Nodal priority through power is also introduced to make WBAN more efficient. Our proposed scheme outperforms IEEE 802.15.6 based CSMA/CA in all major aspects i.e. mean power consumption, network delay, network throughput and network bandwidth efficiency. Thus our proposed scheme has shown significant improvement in IEEE 802.15.6 based CSMA/CA.

\section{References}

[1] Ye, W., Heidemann, J. and Estrin, D. (2002) An Energy-Efficient MAC Protocol for Wireless Sensor Networks. INFOCOM 2002 Twenty-First Annual Joint Conference of the IEEE Computer and Communications Societies, 3, 1567-1576.

[2] Lewis, F.L. (2004) Wireless Sensor Networks. Smart Environments. Technologies, Protocols, and Applications, 11-46. https://doi.org/10.1002/047168659X.ch2

[3] Mahakud, R., Rath, S., Samantaray, M., Sinha, B., Priya, P., Nayak, A. and Kumari, A. (2016) Energy Management in Wireless Sensor Network Using PEGASIS. Procedia Computer Science, 92, 207-212.

[4] Khan, J.Y., Yuce, M.R., Bulger, G. and Harding, B. (2012) Wireless Body Area Network (WBAN) Design Techniques and Performance Evaluation. Journal of Medical Systems, 36, 1441-1457. https://doi.org/10.1007/s10916-010-9605-X

[5] Jovanov, E., Milenkovic, A., Otto, C., De Groen, P., Johnson, B., Warren, S. and Taibi, G. (2006) A WBAN System for Ambulatory Monitoring of Physical Activity and Health Status: Applications and Challenges. 27 th Annual International Conference of the Engineering in Medicine and Biology Society, Shanghai, 17-18 January 2006, 3810-3813.

[6] Varga, A. (1999) Using the OMNeT++ Discrete Event Simulation System in Education. IEEE Transactions on Education, 42, 11 p. https://doi.org/10.1109/13.804564

[7] Nabi, M., Geilen, M. and Basten, T. (2011) MoBAN: A Configurable Mobility Model for Wireless Body Area Networks. Proceedings of the 4th International ICST Conference on Simulation Tools and Techniques, Barcelona, 21-25 March 2011, 168-177. https://doi.org/10.4108/icst.simutools.2011.245511

[8] Demirkol, I., Ersoy, C. and Alagoz, F. (2006) MAC Protocols for Wireless Sensor Networks: A Survey. IEEE Communications Magazine, 44, 115-121. https://doi.org/10.1109/MCOM.2006.1632658

[9] Förster, A. (2011) Implementation of the B-MAC Protocol for WSN in MiXiM. 4th International Workshop to Be Held in Conjunction with SIMUTOOLS. https://summit.omnetpp.org/archive/2011/uploads/slides/OMNeT_WS2011_S5_C1 
_Foerster.pdf

[10] Van Hoesel, L.F. and Havinga, P.J. (2004) A Lightweight Medium Access Protocol (LMAC) for Wireless Sensor Networks: Reducing Preamble Transmissions and Transceiver State Switches. 1st International Workshop on Networked Sensing Systems (INSS), Society of Instrument and Control Engineers (SICE).

[11] Incel, O.D., Van Hoesel, L., Jansen, P. and Havinga, P. (2011) MC-LMAC: A Multi-Channel MAC Protocol for Wireless Sensor Networks. Ad Hoc Networks, 9, 73-94. https://doi.org/10.1016/j.adhoc.2010.05.003

[12] El-Hoiydi, A. and Decotignie, J.D. (2004) WiseMAC: An Ultra Low Power MAC Protocol for Multi-Hop Wireless Sensor Networks. ALGOSENSORS, 4, 18-31.

[13] Hurni, P., Braun, T. and Anwander, M. (2010) Evaluation of WiseMAC and Extensions on Wireless Sensor Nodes. Telecommunication Systems, 43, 49-58. https://doi.org/10.1007/s11235-009-9196-3

[14] Mile, A., Okeyo, G. and Kibe, A. (2018) Hybrid IEEE 802.15.6 Wireless Body Area Networks Interference Mitigation Model for High Mobility Interference Scenarios. Wireless Engineering and Technology, 9, 34-48. https://doi.org/10.4236/wet.2018.92004

[15] Shakir, M., Rehman, O.U., Rahim, M., Alrajeh, N., Khan, Z.A., Khan, M.A., et al. (2016) Performance Optimization of Priority Assisted CSMA/CA Mechanism of 802.15. 6 under Saturation Regime. Sensors, 16, 1421.

https://doi.org/10.3390/s16091421 